\title{
A watertight acrylic-free titanium recording chamber for electrophysiology in behaving monkeys
}

\author{
Daniel L. Adams, ${ }^{1}$ John R. Economides, ${ }^{1}$ Cristina M. Jocson, ${ }^{1}$ John M. Parker, ${ }^{2}$ \\ and Jonathan C. Horton ${ }^{1}$ \\ ${ }^{1}$ Program in Neuroscience and ${ }^{2}$ Laboratory Animal Resource Center University of California, San Francisco, \\ San Francisco, California
}

Submitted 3 May 2011; accepted in final form 9 June 2011

\begin{abstract}
Adams DL, Economides JR, Jocson CM, Parker JM, Horton JC. A watertight acrylic-free titanium recording chamber for electrophysiology in behaving monkeys. J Neurophysiol 106: 1581-1590, 2011. First published June 15, 2011; doi:10.1152/jn.00405.2011.— Neurophysiological recording in alert monkeys requires the creation of a permanent aperture in the skull for repeated insertion of microelectrodes. Most laboratories use polymethyl methacrylate to attach a recording chamber over the skull opening. Here, we describe a titanium chamber that fastens to the skull with screws, using no polymethyl methacrylate. The gap between the base of the chamber and the skull is filled with hydroxyapatite, forming a watertight gasket. As the chamber base osseointegates with the skull, the hydroxyapatite is replaced with bone. Rather than having a finite lifetime, the recording chamber becomes more firmly anchored the longer it is in place. It has a small footprint, low profile, and needs little maintenance to control infection. Toilette consists of occasional application of betadine to clean the scalp margin, followed by application of neomycin, polymyxin, and bacitracin ointment. Antibiotic is also placed inside the chamber to suppress bacterial proliferation. Thickening of the dura within the chamber can be prevented by regular application of mitocycin $\mathrm{C}$ and/or bevacizumab, an antibody against vascular endothelial growth factor. By conducting an e-mail survey, this protocol for chamber maintenance was compared with procedures used in 37 other vision research laboratories. Refinement of appliances and techniques used for recordings in awake monkeys promises to increase the pace of scientific discovery and to benefit animal welfare.
\end{abstract}

primate; macaque; physiology; awake behaving; alert; polymethyl methacrylate; dental acrylic; hydroxyapatite

THE ABILITY TO MAKE DAILY extracellular recordings in alert monkeys has yielded a wealth of valuable data about the function of the primate brain. It has also reduced the number of animals required for neuroscience research, because a single animal can continue to provide data for months or even years. To perform such recordings, two devices are necessary: a stabilizing headpost and a recording chamber. Previously, we described a biocompatible titanium headpost, which attaches with screws alone and requires minimal maintenance (Adams et al. 2007). Here we report a titanium recording chamber, which also attaches with screws and forms a permanent, watertight seal with the skull.

A major advantage of both of these appliances is that they dispense with the acrylic polymer, polymethyl methacrylate (PMMA; also known as Plexiglas, Lucite, Perspex, and Acry-

Address for reprint requests and other correspondence: D. L. Adams, Beckman Vision Center, 10 Koret Way, Univ. of California, San Francisco, San Francisco, California 94143-0730 (e-mail: adamsd@vision.ucsf.edu). lite). PMMA is also known as dental cement, a name that stems from its use in the manufacture of prosthetic dentures and as a "luting agent" to attach prostheses to tooth stumps.

PMMA use in cranioplasty began in Germany (Kleinschmidt 1941; Zander 1963), where it rapidly replaced metal for craniofacial reconstructive surgery following trauma or craniotomy (Sanan and Haines 1997). Lacking adhesive properties, it acts primarily as a filler or grout, and screws are required to anchor it to external bone surfaces like the skull (Nikolis et al. 2009; Smith et al. 1999). In human surgery, PMMA reconstructions are sealed under the scalp, protecting them from infection.

PMMA was first used to secure implanted electrodes in a primate by Delgado (1952). He noted that, "the small hole in the skull is closed with dental cement, which holds the electrodes firmly in position. Only a minute quantity should be used to avoid the formation of an excessive protuberance on the skull." Reporting on a technique for chronic neuronal recording, Sheatz (1961) emphasized that "quantities [of PMMA] should always be minimal, especially in those locations where cement will be adjacent to subcutaneous tissue." In these early experiments, PMMA was used to secure implanted electrodes under the skull for recordings in alert animals. The only exposed appliance was the electrical connector, minimizing the potential for bacterial infection.

To record from multiple sites in the alert animal, one must devise a method to withdraw electrodes and move them to a new location (Wurtz 1968). The first permanent recording chamber was developed by Evarts $(1966 ; 1968)$. It provided an open window to the dura mater to allow repeated electrode penetrations over a small region. A stainless steel cylinder was fastened to the skull with three bolts. The bolt heads were inserted between the bone and dura along slots extending from the trephined skull margin. Nuts were placed on the protruding bolt stems to trap the outer edge of a flange at the cylinder's base. A small amount of dental cement was applied around the base of the cylinder to seal it to the skull.

As Evarts' technique became popular, many laboratories integrated the chamber and the head fixation device in a large dome of PMMA, held in place with slot bolts, bone screws, or machine screws. The covering of a wide area of skull with acrylic is not ideal, for both aesthetic and practical reasons. Bacteria flourish at the margin between the scalp and acrylic, requiring regular cleaning, debridement of granulation tissue, antiseptics, and application of antibiotics. If the seal between the chamber and skull is not perfect, exudate will leak under the implant. The moist interface between implant and skull provides an environment for proliferation of microorganisms. 
Fig. 1. A: lateral view of a Macaca mulatta skull from an animal that developed an infection between the polymethyl methacrylate (PMMA) headcap and the skull. Postmortem revealed extensive thinning of bone that was covered by PMMA. Many screw holes had enlarged sufficiently to release the screws. The two trephine holes were enlarged, with thinned edges. In contrast, excessive bone proliferation was observed at the perimeter of the headcap (arrow). $B$ : view of the chamber interior from inside the skull in another macaque. The base of a stainless steel chamber and the surrounding mass of blue and green PMMA is visible through the trephination. Bone resorption occurred around the chamber base, opening a gap.

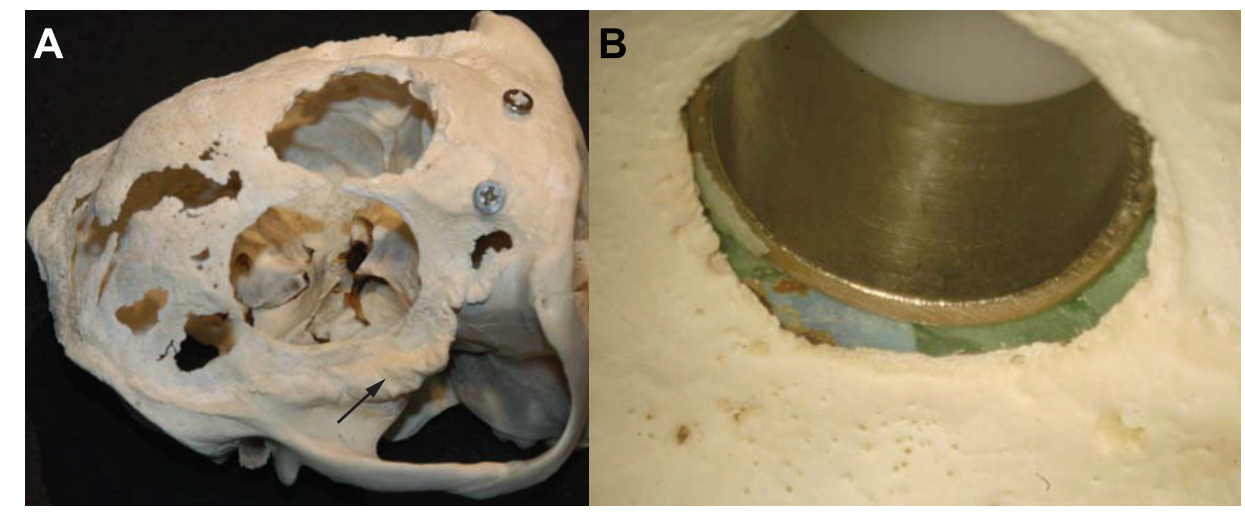

Eventually, infection can spread to the chamber interior, causing a dural abscess or meningitis. Figure 1 shows the skull from a macaque that had two chambers and a headpost implanted for 6 mo in an acrylic headcap. Widespread thinning of the skull occurred from bone resorption, and a large gap developed around the base of each chamber.

The problems associated with acrylic-based implants are familiar, yet this compound remains widely used, perhaps because the known drawbacks of acrylic are feared less than the unknown risks of new materials. Individual laboratories learn to cope with the challenges posed by recording chambers through trial and error; experience is shared largely by word of mouth because few publications address the subject. To facilitate the exchange of information, we surveyed vision research laboratories engaged in daily recordings from alert monkeys to identify key problems associated with recording chambers, and to compare standard operating procedures. Here we review the collective experience of colleagues in the field and describe our development of an acrylic-free, watertight titanium recording chamber designed to reduce skin margin toilette and to promote internal sterility.

\section{METHODS}

The objective was to design, manufacture, and test a recording chamber that attaches securely to the skull and forms a watertight seal with the bone surface. This was achieved by using a titanium chamber fastened to the skull with titanium orthopedic screws. To reduce chronic infection and ensuing bone loss, we opted to eliminate PMMA entirely. Instead, we used hydroxyapatite paste to seal the gap between the base of the chamber and the skull. The chamber was constructed from unalloyed, commercially pure (CP) titanium, because this material is easier to machine than titanium alloys, and its superior ductility permits bending of the feet without fracture. $\mathrm{CP}$ titanium has osseointegrative properties and corrosion resistance in a saline environment, making it suitable for use as a bone-anchored implant (Pohler 2000; Rubo de Rezende and Johansson 1993).

The chamber was machined from a single piece of 2-in.-diameter $\mathrm{CP}$ titanium bar stock. Six evenly spaced feet surrounded the chamber base, each with a single screw hole. The outer surface and base of the chamber were grit-blasted (mesh size 50), roughening the finish to encourage osseointegration (Buser et al. 1991). Three chambers with this design were implanted into male rhesus monkeys, two adult and one juvenile.

Following these three implantations, the design was improved by reducing the number of feet from six to five (Fig. 2). This change was intended to reduce scalp retraction from the chamber wall. The feet separate the scalp from the skull, allowing it to pull back from the chamber. To lower the screw profiles, we ground $1 \mathrm{~mm}$ off their heads before insertion, and countersank the holes more deeply. The weight of the chamber was $12.7 \mathrm{~g}$ (not including the lid or the 5 titanium screws). A digital three-dimensional model is available upon request. The chamber drawing was sent to a computer numerical control workshop for manufacture.

Implantation surgery. All procedures were approved by the University of California, San Francisco Institutional Animal Care and Use Committee. Animals were sedated with ketamine $(10 \mathrm{mg} / \mathrm{kg} \mathrm{im})$ and prepared for sterile surgery. An endotracheal tube was inserted, and ventilation provided with $2 \%$ isoflurane in $50: 50 \mathrm{~N}_{2} \mathrm{O} / \mathrm{O}_{2}$ to maintain surgical anesthesia. ECG, rectal temperature, inspired and expired anesthetic agents, end-tidal $\mathrm{CO}_{2}$, and oxygen were monitored continuously. The animal was placed on a heated blanket in a stereotaxic apparatus. The scalp was shaved and swabbed with $5 \%$ povidoneiodine. An important factor in promoting good wound closure and long-term implant health is to avoid circulatory compromise of the scalp. Care was taken to preserve the major tributaries of the occipital arteries and the superficial temporal arteries by tracing their course with a miniature ultrasonic Doppler flow detector (model 811-BTS, Parks Medical Electronics, Aloha, OR).

To implant the chamber over the operculum of striate cortex, an incision was made starting at the midline, extending $\sim 40 \mathrm{~mm}$ posterior laterally, avoiding the arteries identified by ultrasound. Bone was
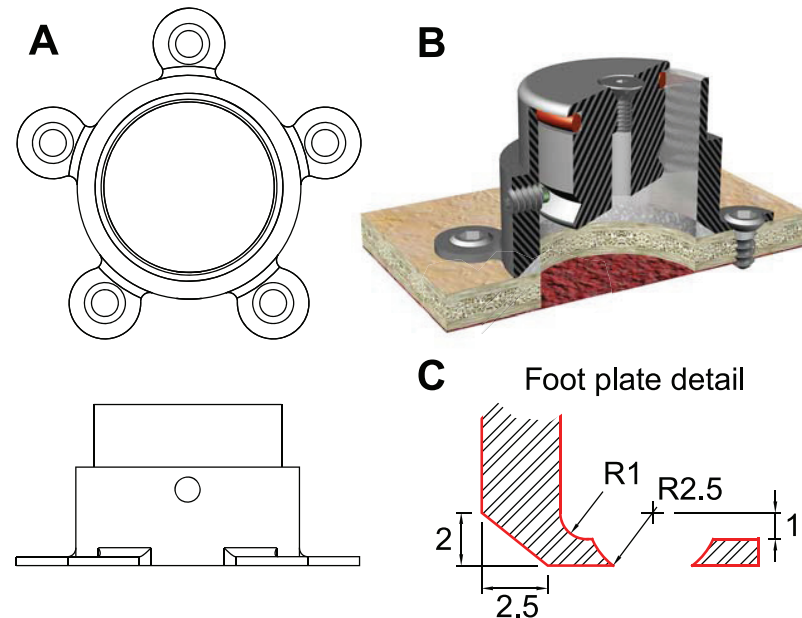

Fig. 2. A: orthographic drawing of the acrylic-free chamber. Inner diameter of the chamber bore is $19 \mathrm{~mm}$. $B$ : rendering of cross section through the chamber and lid mounted on a trephined bone surface. The cross-sectioned lid has been rotated slightly to show the silicone o-ring (orange) and the $\mathrm{V}$-shaped groove that mates with a nylon-tipped set screw to hold the lid in place. The lid's vent is shown, sealed with a countersunk screw. A ring of hydroxyapatite seals the chamber to the skull. $C$ : detail cross section of the chamber base, showing the $2 \times 2.5 \mathrm{~mm}$ chamfer that is packed with hydroxyapatite following implantation. The screw hole is countersunk with a spherical profile of radius $2.5 \mathrm{~mm}$ to match the shape of "Synthes style" bone screw heads. 
exposed over a region measuring $\sim 30 \times 30 \mathrm{~mm}$ by elevating the galea and periosteum. It is sometimes necessary to disinsert the temporalis muscle from the superior temporalis line to widen the skull exposure. This is done best with a small bone curette to avoid bleeding and muscle damage.

The chamber was matched to the curvature of the skull by placing it in a vise and gently bending the feet. It was immersed in a sterilizing solution before placing it back on the skull to test the fit. Once the feet were curved suitably to conform to the skull surface (maximum $\sim 1$ $\mathrm{mm}$ gap between skull surface and underside of foot), a 2-mm orthopedic drill-bit (part no. 2.0 QCK, Veterinary Orthopedics Implants, South Burlington, VT) was used to make a pilot hole (Fig. 3A). To prevent accidental perforation of the dura, we sheathed the drill bit in 12-G hypodermic tubing. A series of tubes were precut in lengths ranging from 2 to $5 \mathrm{~mm}$ shorter than the drill bit so that the maximum
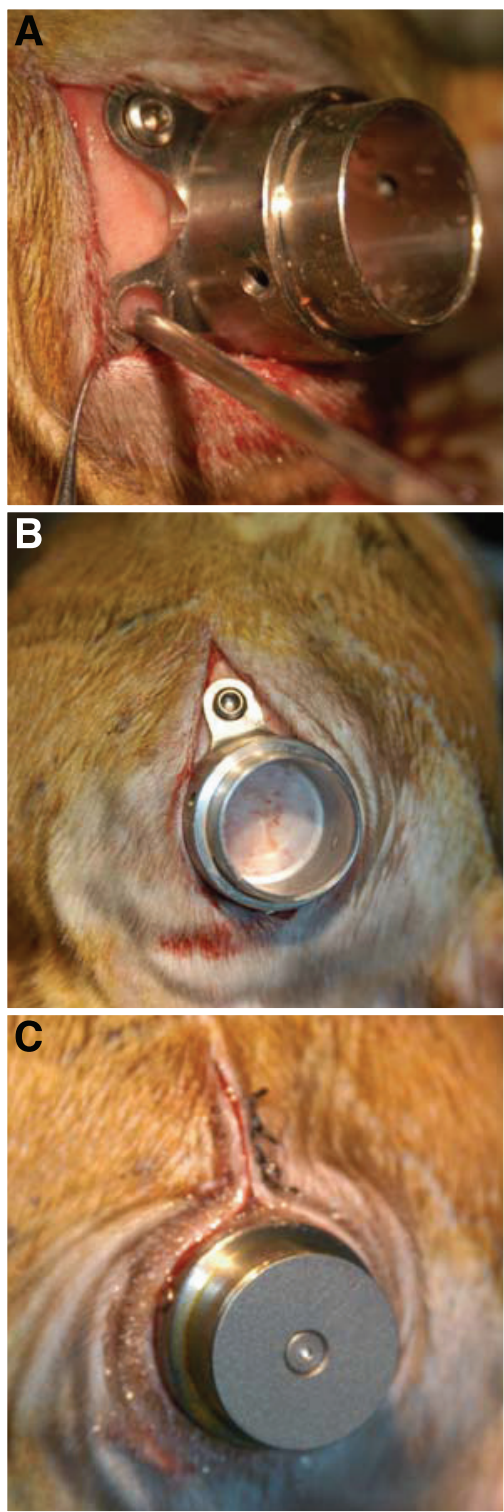

Fig. 3. Chamber implantation procedure. $A$ : one screw has been placed, and the pilot hole for the second is being drilled. The scalp is retracted with a muscle hook, and the drill bit is sheathed in a $12-\mathrm{G}$ tube that leaves a limited length of drill bit exposed. Holes are drilled slowly by hand while holding the drill in a tap-wrench. $B$ : once all screws are in place but prior to trephination, the hydroxyapatite gasket is formed. It is visible as a white ring around the floor of the chamber. $C$ : following implantation, the scalp is sutured tightly around the sealed chamber. depth of the hole could be limited precisely. The screws were 2.7 mm-diameter $\times 8 \mathrm{~mm}$-long titanium cortex screws (part no. T270.08, Veterinary Orthopedics Implants). Further details have been published previously (Adams et al. 2007).

Once all the screws were in place, the chamber was sealed to the skull surface using hydroxyapatite paste. Mimix QS (Biomet Microfixation, Jacksonville, FL) is a fully synthetic apatite calcium phosphate material formed by tetracalcium and tricalcium phosphate. Supplied as a sterile, nonpyrogenic, hydrophilic white powder, it is mixed with citric acid to form a moldable paste. It was mixed and pushed into the circular cavity between base of the chamber and the bone surface using a spatula. A gloved finger was swept around the ring to push the paste further into the gap, forming a smooth-surfaced hydroxyapatite gasket. This maneuver must be performed quickly because the paste begins to set after only 2-3 min. When complete, the paste formed a continuous bead, caulking the space between the chamfered chamber base and the bone surface (Fig. 3B).

After irrigation with physiological saline, the galea was sutured over the top of the chamber feet using 5-0 or 6-0 polyglactin (Vicryl, Ethicon, Somerville, NJ) absorbable suture. The skin incision was closed with 4-0 silk, drawing the skin tightly up to the chamber wall. It was necessary to suture only on one side of the chamber; the scalp incision on the other side abutted the chamber wall (Fig. 3C). Topical antibiotic ointment was applied to the incision (neomycin sulphate, polymyxin sulfate and bacitracin zinc, Vetro-biotic, Pharmaderm, NY). The chamber was filled with the antibiotic ointment prior to closing the lid. After surgery, an opiate analgesic was administered every $8 \mathrm{~h}$ for 2 days (buprenorphine $\mathrm{HCl}, 0.3 \mathrm{mg} / \mathrm{kg} \mathrm{im}$ ).

Trephination. In one animal, the skull was trephined at the time of chamber implantation, while in two animals the procedure was carried out weeks later. The delay was intended to allow bone remodeling to reinforce the screws, so that the chamber was securely fastened before trephination. It also provided extra time for the hydroxyapatite seal to mature before opening the skull.

Using the anesthetic technique described above, a circular disk of bone was removed using a $19 \mathrm{~mm}$ (3/4 in) diameter carbide grit hole saw (Lenox Tools item no. 2991212CG). We modified the hole saw by removing the abrasive grit from its outer surface with a grinding wheel to prevent abrasion of the inside wall of the chamber. Since the hole saw was guided by the chamber alone, its central drill bit was withdrawn. A standard variable-speed electric drill was used to cut out the bone disk. Rotation speed was kept below $200 \mathrm{rpm}$, and the chamber interior was flushed frequently with sterile saline to minimize heat buildup and to remove bone dust. Periodic checking of the cutting progress allowed us to bias the pressure on different quadrants of the circular cut to achieve an even trephination. Inevitably, however, one portion of the circular hole reached the dura first. The advantage of using an abrasive hole saw, rather than a tool with sharp teeth is that the dura is pushed away by the cutting surface and not damaged.

After the skull disk was removed, the chamber interior was allowed to dry until the dura became translucent. This made it possible to identify the lunate sulcus, and therefore to know the approximate locations of V1, the V2 border, and V4 (Fig. 4). Photographs were taken so that future electrode penetrations could be targeted to the desired cortical area, and to avoid puncturing vessels in the lunate sulcus. The chamber was then refilled with antibiotic ointment, and the lid was closed. After surgery, buprenorphine was administered as described above.

Assessment of performance. After 16 mo of daily extracellular recordings, fluoroscopy was performed in one animal to evaluate the seal between the chamber and skull (Phillips Allura Xper FD-10). Images were obtained before and $45 \mathrm{~min}$ after filling the chamber with a liquid contrast agent (lohexol 350, Omnipaque, GE Healthcare). The next day the animal was euthanized for histological analysis. A lethal dose of pentobarbital sodium $(150 \mathrm{mg} / \mathrm{kg}$ iv) was administered, followed by perfusion with normal saline and $1 \%$ paraformaldehyde. 


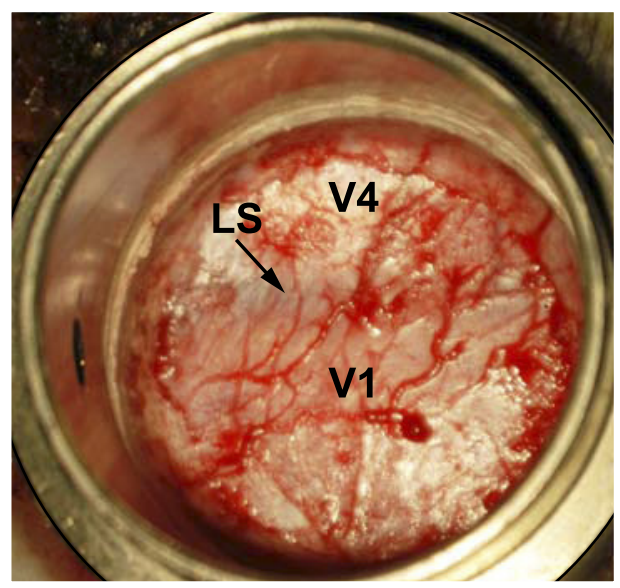

Fig. 4. Chamber interior immediately following trephination, 2 wk following chamber implantation. When allowed to dry slightly, the dura becomes semitransparent, and the large blood vessels that run along sulci can be visualized. Photographs taken at this point are useful for planning electrode penetrations to avoid surface vessels. V1, striate cortex; LS, lunate sulcus; V4, visual area 4.

The skull cap was removed, with dura and chamber attached. After decalcification, thin sections of bone were stained with hematoxylin and eosin to assess the bone/titanium interface and the hydroxyapatite gasket.

\section{RESULTS}

The recording chamber can be implanted at the same time as the headpost, or in a subsequent surgical procedure. Simultaneous implantation has the advantage of sparing the animal a second operation. However, if the monkey will require a prolonged training period before recordings, it may be preferable to install the headpost first.

Previously, we described a titanium headpost with a footplate that attaches to the cranium without acrylic. A survey revealed that 3/107 titanium headposts of similar design failed

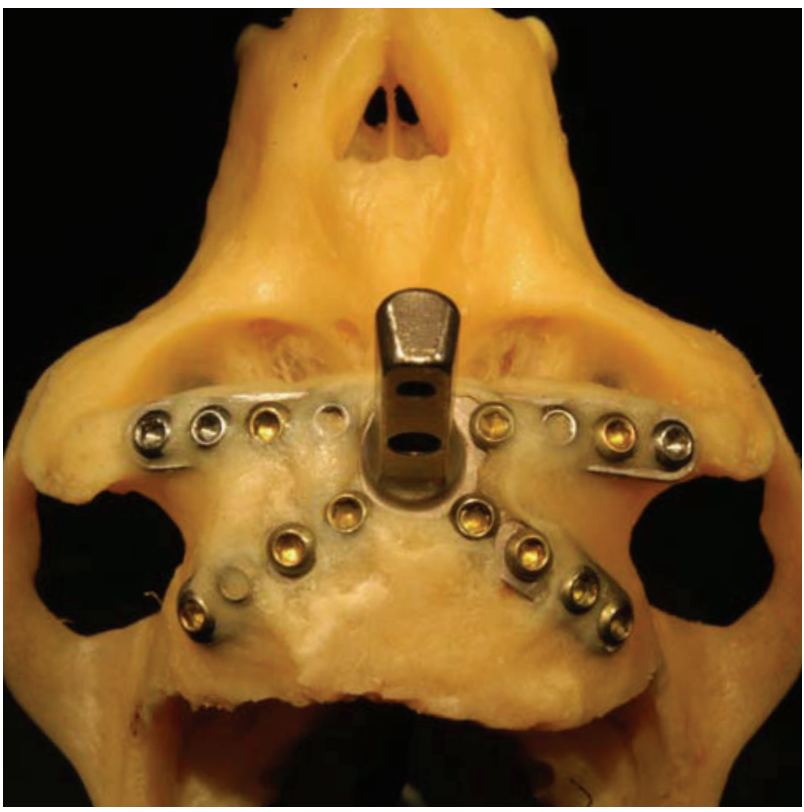

Fig. 5. Postmortem view of titanium headpost implantation in an adult macaque. The headpost was in place for $3 \mathrm{yr}$ and $8 \mathrm{mo}$. because the footplate fractured (Adams et al. 2007). As a precaution, our headpost design has been modified by increasing the thickness of the footplate from 1.0 to $1.5 \mathrm{~mm}$. Other minor improvements have also been made (contact authors for details). This headpost osseointegrates with the skull, forming a permanent attachment (Fig. 5).

The recording chambers we designed were implanted in three monkeys, and used for $6 \mathrm{mo}, 16 \mathrm{mo}$, and $4 \mathrm{yr}$. After implantation, photographs were taken at regular intervals to assess performance (Fig. 6A). Wound healing was rapid, with the circular scalp margin initially remaining apposed to the chamber wall, covering the feet and screws entirely. With time, scalp retraction sometimes occurred over chamber feet (Fig. $6 B)$. To prevent scalp retraction, the chamber design was modified slightly (Fig. 2). It is preferable for the scalp to cover the feet, but exposure of the chamber feet did not cause screw attachment to the skull to loosen. With normal fur regrowth, the chambers appeared unobtrusive.

Chamber performance. The chamber lid was designed to fit inside, rather than outside the chamber bore, to give it a flush profile. A silicone o-ring in a circumferential groove in the lid provided a watertight seal against the chamber bore. A vent hole in the center of the lid that sealed with a countersunk machine screw allowed excess liquids (e.g., antibiotic ointment) to escape from the chamber during lid insertion.
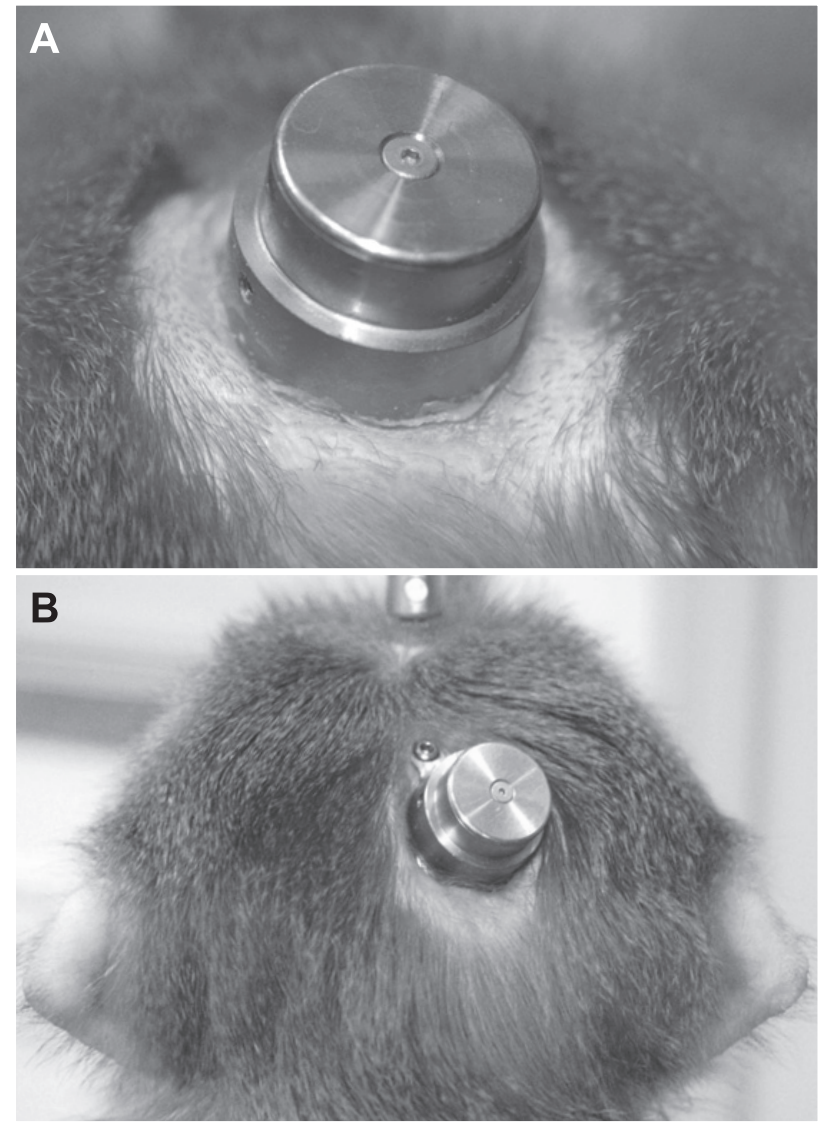

Fig. 6. A: view of the chamber 16 mo after implantation. Fur surrounding the chamber has been trimmed. $B$ : animal in Fig. 3, photographed $4 \mathrm{yr}$ after implantation. The scalp has receded from the chamber, exposing the anterior foot and its screw head. However, the scalp remains healthy with scant exudate along the margin around the chamber. 


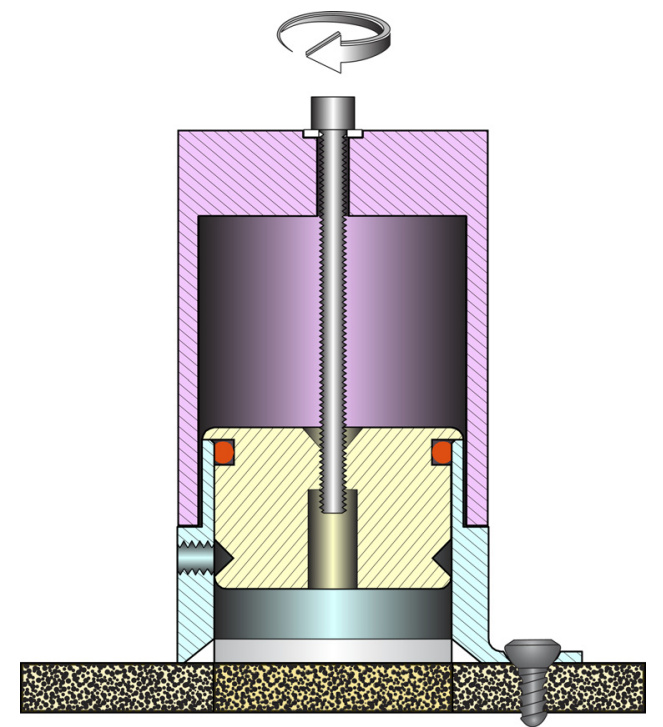

Fig. 7. Cross section of the chamber and a tool for removal of the lid. The tool resembles an inverted cup with a hole for a freely turning screw in the center of its base. A Teflon washer (white) is placed under the screw head. A stubborn lid can be quickly and easily removed as follows: the retaining set screw and vent screw are removed, and the extraction tool (magenta) is placed over the chamber (cyan). The tool rests on the chamber's shoulder. A socket head cap screw is placed through a clearance hole at the tool's center and into the threaded vent hole of the lid (yellow). Rotation of the screw pulls the lid out of the chamber bore without straining the chamber or headpost attachments to the skull.

If not opened regularly, the lid sometimes became difficult to remove from the chamber. Pulling hard on the lid when the animal is stabilized is not advisable because it stresses both chamber and headpost attachments. A lid extraction tool that exploited the thread of the vent hole was invented to pull the lid into a cup-shaped chamber (Fig. 7). It pushed on the shoulder of the chamber's outer wall, applying no force to the bone screws. Lid adhesion occurs because fluid dries between the chamber bore and lid; lubrication with antibiotic ointment prior to lid replacement helps to avoid this problem.

The lid was secured by a $4-40$ stainless steel set screw, tipped with nylon to avoid marring the surface. The screw tip inserted into a V-shaped groove, pulling the lid down to its fully seated position when tight. With daily tightening and loosening, these retaining set screws were prone to wear. If the hexagonal cup became rounded, removal of a tightened set screw was difficult. Galvanic corrosion, resulting from contact between two different metals in a saline environment, also caused the stainless steel set screws to deteriorate. Using titanium screws would prevent this. To avoid difficulty in removing the set screws, they were replaced monthly.

A second function of the retaining set screw was to hold in place a plastic chamber insert containing a square grid of 1.0-mm-spaced holes (Crist Instrument, Hagerstown, MD). Before a recording session was started, the grid was placed into the chamber, aligned with a fiduciary mark on the chamber rim, and locked in place with the set screw. Electrodes were inserted through grid holes to maintain a Cartesian coordinate system of recording sites that was consistent from one day to the next.

When recording from surface cortex, it is preferable to penetrate the dura with a bare electrode to avoid damage from a metal guide tube. However, the dura inside the recording chamber becomes abnormally thick with time, making it resistant to puncture. Electrodes are frequently damaged because the shaft buckles from the compressive force necessary to penetrate the dura. To overcome this problem, we employed a system of concentric guide tubes to eliminate electrode buckling. It permitted fragile glass-insulated tetrodes (Thomas Recordings, Geissen, Germany) to penetrate the dura (Fig. 8).

Watertight chamber seal. A principal feature of the chamber's design was that it resulted in a watertight seal with the skull surface. This seal prevented the escape of fluid from the chamber and served as a barrier to infection. To test the seal formed by the hydroxyapatite gasket, we obtained fluoroscopic

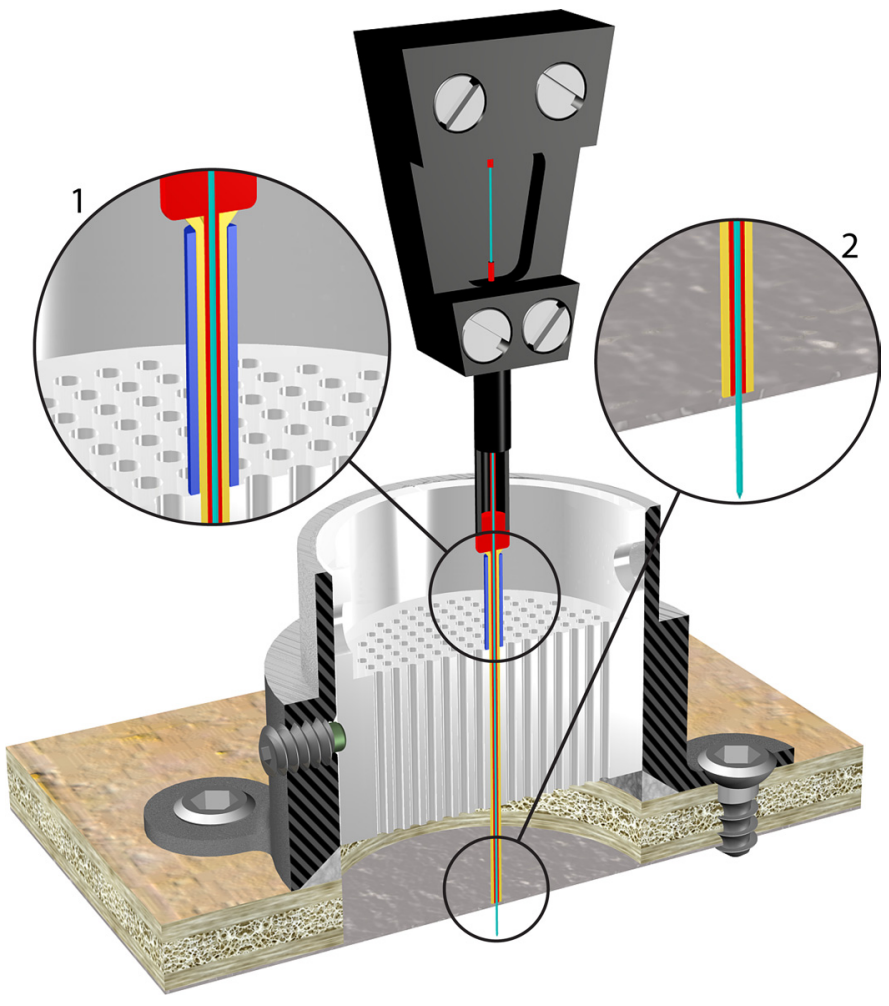

Fig. 8. System to provide axial support to prevent buckling of glass-insulated electrodes as they penetrate dura. A schematic cross section of the chamber and grid insert (Crist Instrument, Hagerstown, MD) is shown. The procedure for electrode penetration is as follows. Remove the lid and place liquid agarose (Sigma A0169) at $38^{\circ} \mathrm{C}$ inside the chamber. Immediately insert the grid so that excess agarose is displaced through the grid holes, and the entire chamber between the bottom surface of the grid and the dura is filled. Align the grid with a fiduciary on the chamber rim. Tighten the set screw to hold the grid in place. Insert the blunt 23-G outer guide tube (yellow) into the selected grid hole and gently push it down, through the agarose, until it contacts the dural surface. When gently pressed against the dura, the tube will "bounce back" to a constant depth. Using a graduated periodontal probe, measure the length of the tube that protrudes from the grid. Select a spacer (blue) of the same length $( \pm 0.5 \mathrm{~mm})$ from a set of precut $19-\mathrm{G}$ tubes. Remove the $23-\mathrm{G}$ guide tube, pass it through the spacer, and reinstall it into the grid. The flared end of the $23-G$ guide tube (inset 1 ) will prevent it from passing through the spacer and advancing any deeper. As a result, the tip of the guide tube will be held in a stable position against the surface of the dura (inset 2). The electrode (cyan) is sheathed inside a blunt $31-\mathrm{G}$ tube (red) that is attached to the micromanipulator. This $31-\mathrm{G}$ tube is the same length as the $23-\mathrm{G}$ guide tube. Align the 31-G tube using the $X / Y$ stage and insert it into the $23-G$ guide tube. Now, the electrode can be driven out of the $31-G$ tube. Because it is supported axially, it cannot buckle, and its sharp tip will penetrate thickened dura. Furthermore, the agarose will prevent lateral movement of the guide tubes and minimize "tenting" of the dura that could otherwise result in electrode fracture. 
Fig. 9. Fluoroscopic images of an acrylic-free chamber taken to test the seal between chamber and skull. $A$ : side view taken prior to filling the chamber with contrast agent. $B$ : top view with $5 \mathrm{ml}$ of contrast agent in the bore. The contrast agent was left in the chamber for $45 \mathrm{~min}$ and then removed. $C$ : high-sensitivity (shorter exposure) image taken immediately after removal of the contrast agent. There is no evidence of leakage. $D$ : side view after contrast agent removal. Seepage of the agent between the scalp and the skull would be visible as a dark line in this image but not in $A$.
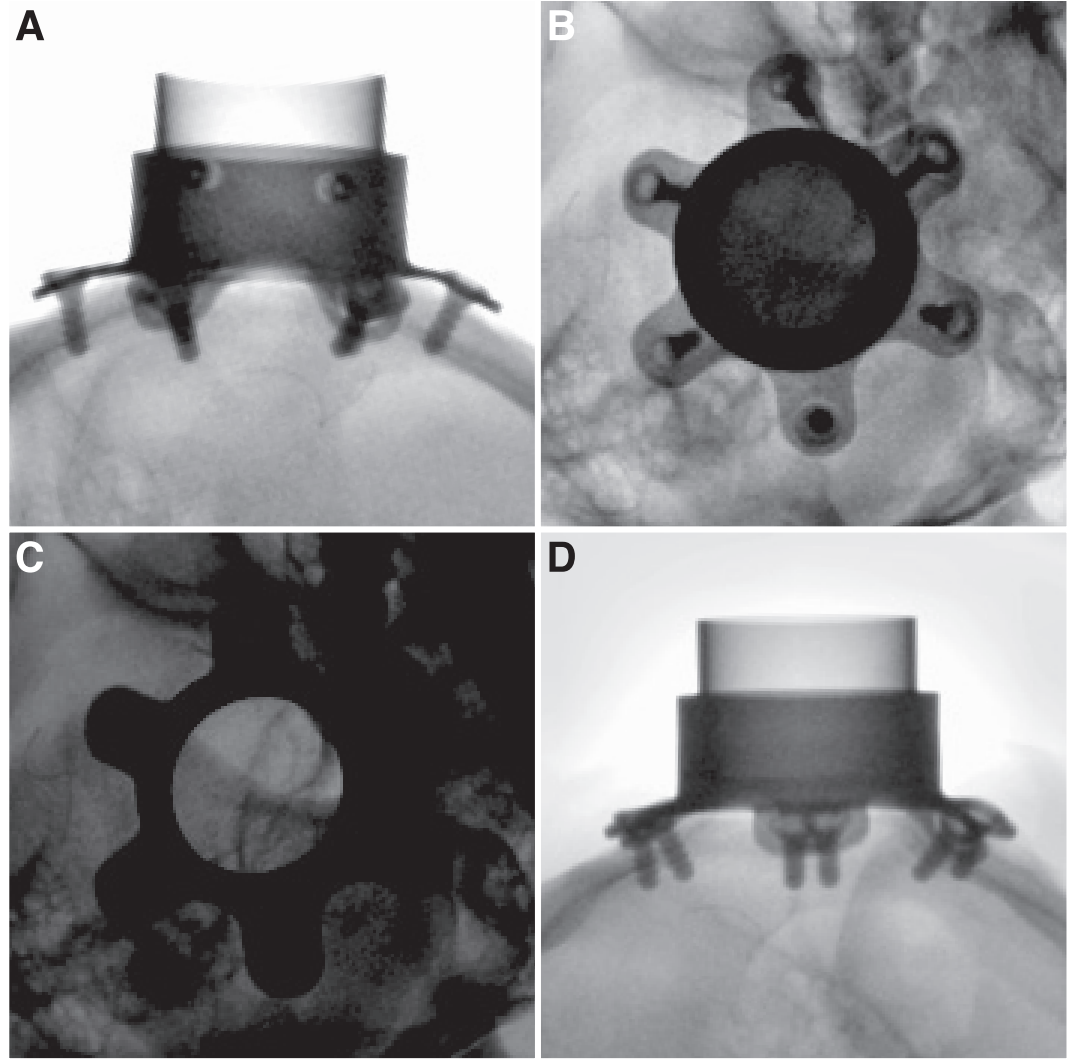

images of a chamber that had been implanted for 16 mo. The chamber was filled with $5 \mathrm{ml}$ of a radiopaque contrast agent. There was no leakage of the contrast agent into the subdural or epidural space. More importantly, there was no extravasation of contrast agent under the scalp surrounding the chamber, even after 45 min (Fig. 9). This indicated that the seal between the chamber base and the skull was watertight.

Bacterial colonization. The scalp margin around the chamber was cleaned as needed (usually weekly) by gentle rubbing with a betadine swab, followed by application of a triple antibiotic ointment consisting of neomycin, polymyxin, and bacitracin. A dab of the antibiotic ointment was also placed inside the chamber after every recording session to maintain sterility. Surveillance cultures for aerobic, anaerobic, and fungal organisms were performed periodically from the scalp margins and chamber interior in all three animals. The scalp cultures always grew Staph aureus, despite weekly application of antibiotic. The organism was sensitive to bacitracin, oxacillin, cephalothin, vancomycin, sulfisoxazole, timethoprim, tetracycline, tobramycin, ciprofloxacin, and moxifloxacin and resistant to erythromycin. In one animal, cultures were also positive for corynebacterium, a gram-positive rod found in the mucosa and normal skin flora of primates.

In two animals, the chamber was not opened for several weeks, during an interruption in recordings. Cultures were taken immediately upon reopening the chamber, to see if the interior remained sterile. In both cases, the cultures were positive for Staph aureus. Neomycin, polymyxin, and bacitracin antibiotic ointment was placed inside the chamber, and it was recultured 3 days later, with negative results.

Control of dural proliferation and neovascularizaton. Removal of a disk of skull to allow access for electrode record- ings induces a proliferative response in the dura. The dura becomes progressively thickened and vascularized, making it more difficult to penetrate and prone to bleeding. An antimitotic agent, 5-fluorouracil (5-FU), has been used to inhibit this process (Spinks et al. 2003). We have tested mitomycin C and bevacizumab, two other compounds with the potential to alleviate the problem of dural thickening.

Mitomycin C is a potent DNA cross-linker, giving it antineoplastic and antibiotic properties. It is used intravenously for chemotherapy and topically in eye surgery to limit scar tissue formation. In one animal, we tested mitomycin $\mathrm{C}$ to prevent tissue proliferation inside the chamber. To ensure even application and to prevent spillage, we placed a disk of gauze on the chamber floor and soaked it with mitomycin C $(1 \mathrm{mg} / \mathrm{ml})$. After $10 \mathrm{~min}$, the gauze was removed, and the chamber was irrigated with sterile saline. Mitomycin C was applied weekly for $3 \mathrm{mo}$, and then monthly for another $3 \mathrm{mo}$. The dura remained thin, avascular, and had a smooth, pale appearance. During this period, daily recordings were made from single cells in the operculum of V1 using glass-insulated tetrodes without any obvious adverse effects from mitomycin C.

An alternative approach to prevent dural proliferation is to use an agent that suppresses neovascularization. Bevacizumab (Avastin, Genentech/Roche), an antibody against vascular endothelial growth factor, was tested in one animal. After being sealed for several months, during a hiatus in recordings, the dura appeared thickened and vascularized (Fig. 10A). Bevacizumab (5 mg in $0.2 \mathrm{ml}$ ) was applied twice. A week later, granulation tissue could be removed easily with minimal bleeding by wiping the dura with a cotton swab (Fig. 10B). Weekly treatment with bevacizumab kept the dura soft and thin, so that 


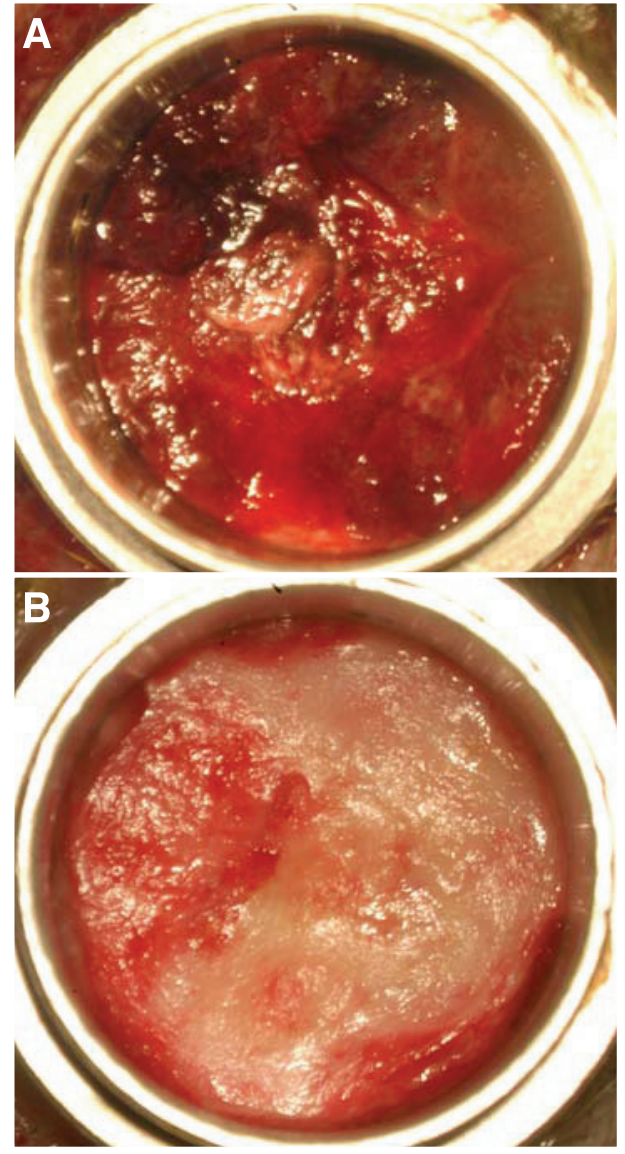

Fig. 10. Assessment of bevacizumab to reduce vascularization of dura and granulation tissue in the chamber. A: photograph of chamber interior following a period of $>3$ mo without removal of the lid. $B$ : the same chamber interior 1 wk after applying two $0.5-\mathrm{mg}$ doses of bevacizumab in $0.2 \mathrm{ml}$ on consecutive days. The dura mater was wiped gently with a sterile Q-tip to remove loose tissue, causing little bleeding.

it could be penetrated daily with glass-insulated tetrodes. There was no apparent effect on the quality of recordings.

Bone remodeling. Necropsy was performed 16 mo after implantation in one monkey. New bone growth had partially covered some of the feet and retaining screws (Fig. 11A). The base was well opposed to the skull, with no gap (Fig. 11B). After removal of the chamber, it was evident that the skull had remodeled to form a perfect impression of the chamber base. The chamfer cavity and gaps between the chamber feet and skull were filled completely by bone (Fig. $11 \mathrm{C}$ ). Even the imprint of the $\sim 50-\mu \mathrm{m}$ groves on the titanium surface, resulting from the manufacturing process, was visible on the bone surface (Fig. 11D). Histological examination of the decalcified specimen showed that the original hydroxyapatite gasket was replaced by new bone tissue, resulting in intimate contact between the chamfered base of the chamber and the remodeled skull surface (Fig. 12). The new growth had the typical characteristics of healthy lamella bone.

A lip of newly formed bone, up to $3 \mathrm{~mm}$ wide, had grown from the edge of the original trephination margin into the chamber interior (Fig. 11B). This is a normal healing response and signifies that the bone underneath the implant was healthy. Eventually, bone regrowth limits the area of brain available for neural recording. If necessary, this problem can be solved by reboring the chamber. If no further recordings are planned, one can let the skull grow back to close the trephination site entirely.

Survey of recording chambers. To share experience among different laboratories, we emailed a questionnaire to 65 US investigators using recording chambers for vision research in alert monkeys. Replies were received from 37 investigators. All but one reported using dental acrylic for recording chambers. The majority (33/36) used the traditional approach, embedding both the chamber(s) and headpost in a single acrylic headcap. Three had adopted an acrylic-free technique to attach headposts, but used acrylic to anchor the chamber to nearby screws and to seal gaps between the chamber base and skull. Most investigators stated that controlling infection and dural thickening were the most challenging problems associated with chamber implants.

A number of antiseptic agents were applied on a regular basis to control infection at the skin margin around the implant. The most common substance was povidone-iodine (Betadine), used by 24 investigators. Another 13 investigators used chlorhexidine (Nolvasan). Other antiseptic agents used to control skin margin infection were as follows: silver nitrate, dilute hydrogen peroxide, Dakin's solution, benzalkonium chloride, trypsin (Granulex), $1 \%$ iodine in ethylene glycol and propylene glycol (Xenodine), ichthammol, and isopropyl alcohol. In addition to antiseptics, 16 investigators regularly applied topical antibiotics to the skin margins. Neomycin sulfate, polymyxin sulfate, and bacitracin zinc ointment were used by nine investigators. Other antibiotics included the following: Neo-Predef (a combination of neomycin and isoflupredone acetate and tetracaine), nitrofurazone, and chloramphenicol.

Some investigators expressed doubt about the wisdom of using antiseptics and antibiotics inside recording chambers because of potential cerebral toxicity. Nonetheless, 19 investigators used an antiseptic, sometimes followed by sterile saline irrigation. Povidone-iodine was used by seven investigators and chlorhexidine by three investigators. Other agents mentioned were as follows: chlorine dioxide (Clidox), sodium hypochlorite, and Dakin's solution. Antibiotics were used inside the chamber by 17 investigators. Neomycin sulfate, polymyxin sulfate, and bacitracin zinc ointment were used by the majority. Other antibiotics mentioned were as follows: Maxitrol (neomycin-polymyxin-dexamethasone), gentamicin, and chloramphenicol.

To avoid dural thickening, five investigators used 5-FU, and one used mitomycin-C. There were no reports of adverse effects from these agents. No investigator reported experience with bevacizumab.

\section{DISCUSSION}

The use of implanted chambers to allow daily access to the brain for extracellular recordings in alert monkeys poses many challenges for the investigator. These devices provide a portal for introduction of microelectrodes, but they also breach the brain's defenses. In essence, an animal with an implanted chamber has a permanent transcranial fistula, which requires regular wound care to suppress the proliferation of infectious agents. The removal of skull by trephination induces a chronic exudate from the surface of the exposed dura inside the chamber. This exudate often becomes mixed with cerebrospinal fluid, which drains through perforations created by guide 
Fig. 11. Postmortem examination of the titanium/ bone interface in a monkey implanted for $16 \mathrm{mo}$. $A$ : high-magnification photograph of one chamber foot, showing the extent of new bone growth around and over the metal. $B$ : view inside the bore of the chamber, focused at its base. A ridge of new bone (arrow) has covered the circular face of the trephination to extend inside the chamber bore. $C$ : the chamber has been removed, and the outer skull surface photographed. The impression of the chamber's foot print is clearly evident because bone has grown to close the gap between chamber and skull. $D$ : high-magnification view of the region outlined with a white box in $C$. The bone surface is textured with concentric 50$\mu \mathrm{m}$-wide grooves. These are an imprint of the machined metal surface. Inset shows the surface of the chamber's circular chamfer at the same magnification.
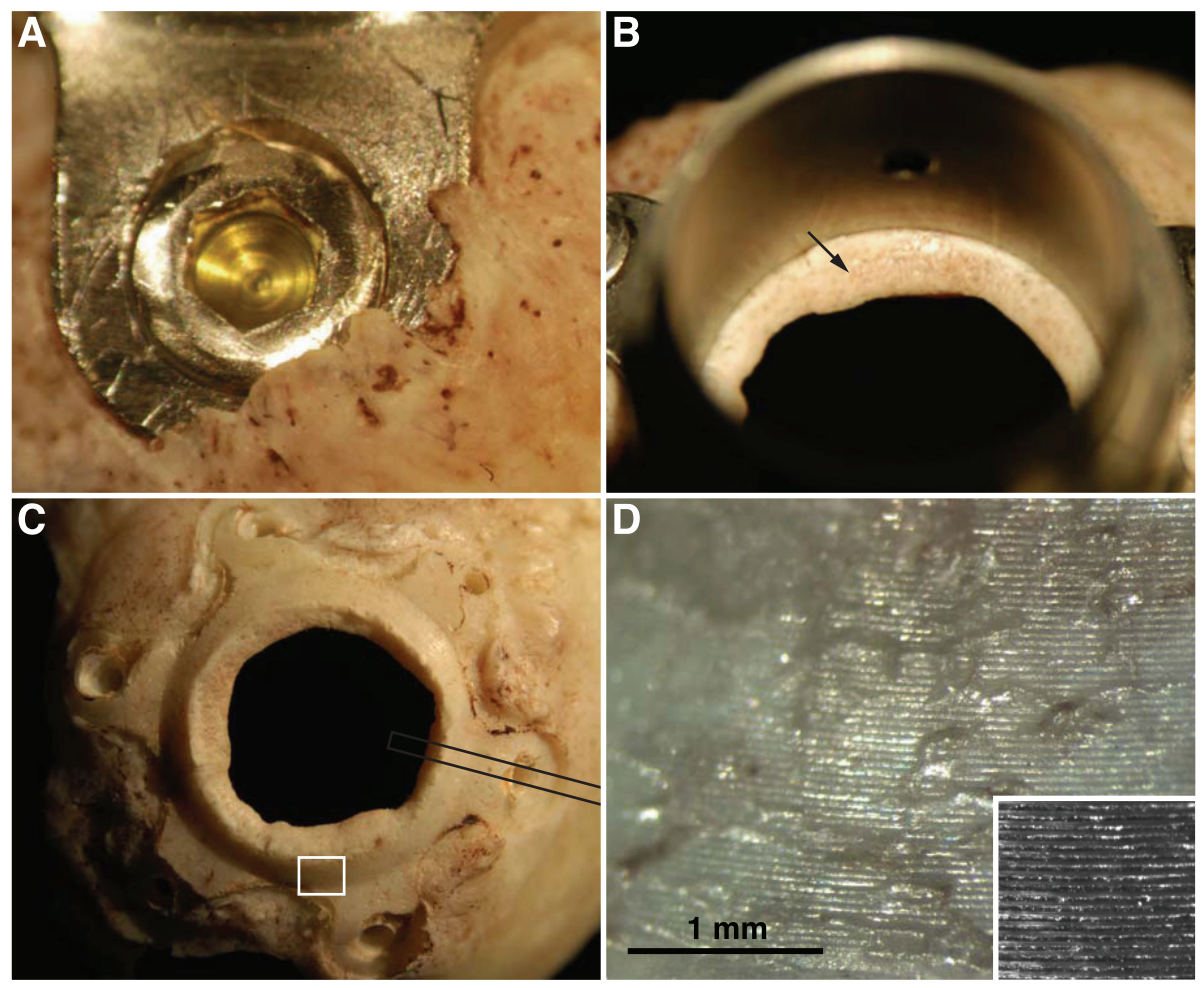

tubes and microelectrodes. A suppurative discharge can leak between the chamber and skull, providing a conduit for entry of infectious organisms from the scalp surface. This creates a risk of meningitis, cerebritis, abscess, and even death.

PMMA was initially used in acute and short-term experimental implants in small quantities as a sealant to close the gap between the chamber and the skull (Evarts 1966; Evarts 1968; Sheatz 1961). As the mechanical and technical demands of implanted devices increased, it was used in greater amounts and took on an additional structural role. However, its failure to bond permanently to the skull surface can result in the ingrowth of soft tissue and the loss of a watertight seal. Fluid seeps along the interface between the headcap and skull, providing a moist, sequestered environment for growth of bacteria and fungi. The common skin flora that colonize this habitat are potent stimulators of bone resorption (Nair et al. 1995). Weakening and loss of bone tissue surrounding the metal fasteners that anchor the acrylic cap to the skull can result eventually in implant detachment.

Recognizing the drawbacks of PMMA, Logothetis (2002) has pioneered the use of biocompatible acrylic-free implants. Several physical and chemical properties combine to make titanium the most suitable material for implantable biomedical devices. A protective oxide layer renders titanium's surface inert, making it compatible with biological tissue. Contact between titanium and bone promotes the formation of a direct interface, with no intervening soft tissue (Shi et al. 2008). This process, known as osseointegration (Brånemark et al. 1969), allows the apposing bone to proliferate so that the titanium screws and footplates that anchor the implant become embedded in the skull. The use of a screw-on titanium chamber also diminishes the size and profile of the implant, making it less obtrusive and unsightly. Without an acrylic headcap, more scalp is preserved, leaving the skull mostly covered by normal, healthy tissue. Bringing the scalp up to the chamber wall reduces the exposed margin of surrounding tissue, thereby reducing the burden of chronic wound infection. Colonization of the scalp margin by infectious organisms can be controlled by direct application of topical antiseptics and antibiotics. With acrylic, organisms may proliferate underneath the headcap, where they are inaccessible to antiseptics and antibiotics.

We used hydroxyapatite, instead of PMMA, to seal the small gap between the chamber base and skull surface (Fig. 3B). Hydroxyapatite supports bone proliferation and is replaced eventually by living bone matrix (Fig. 12). As a result, the chamber and skull became apposed intimately, forming a stable seal. Fluoroscopy with a liquid contrast agent proved that the seal was watertight, preventing leakage of chamber contents under the scalp.

Despite the tight seal between chamber and skull, S. aureus grew inside the chamber, unless antibiotics were administered regularly. On occasions when daily recordings were suspended and the chamber was left unopened, cultures were positive for $S$. aureus. This bacterium is $\sim 0.5 \mu \mathrm{m}$ in diameter, small enough to pass easily through the helical channel between a screw and its threaded hole. We suspect that bacteria can enter the chamber via the set-screw hole in the chamber wall. Addition of a compressible washer or an o-ring may seal this potential leak to allow a closed chamber to remain sterile indefinitely.

Staphylococci and corynebacteria were cultured repeatedly from the scalp margins surrounding the recording chambers. These organisms are normal primate skin flora (Kluytmans et al. 1997). Their growth can be suppressed by applying antiseptics and antibiotics to the wound margin, but they cannot be eliminated entirely (Owens and Stoessel 2008). S. aureus is a potential pathogen: it is the most common cause of osteomyelitis and orthopedic implant infection in humans (Moyad et al. 


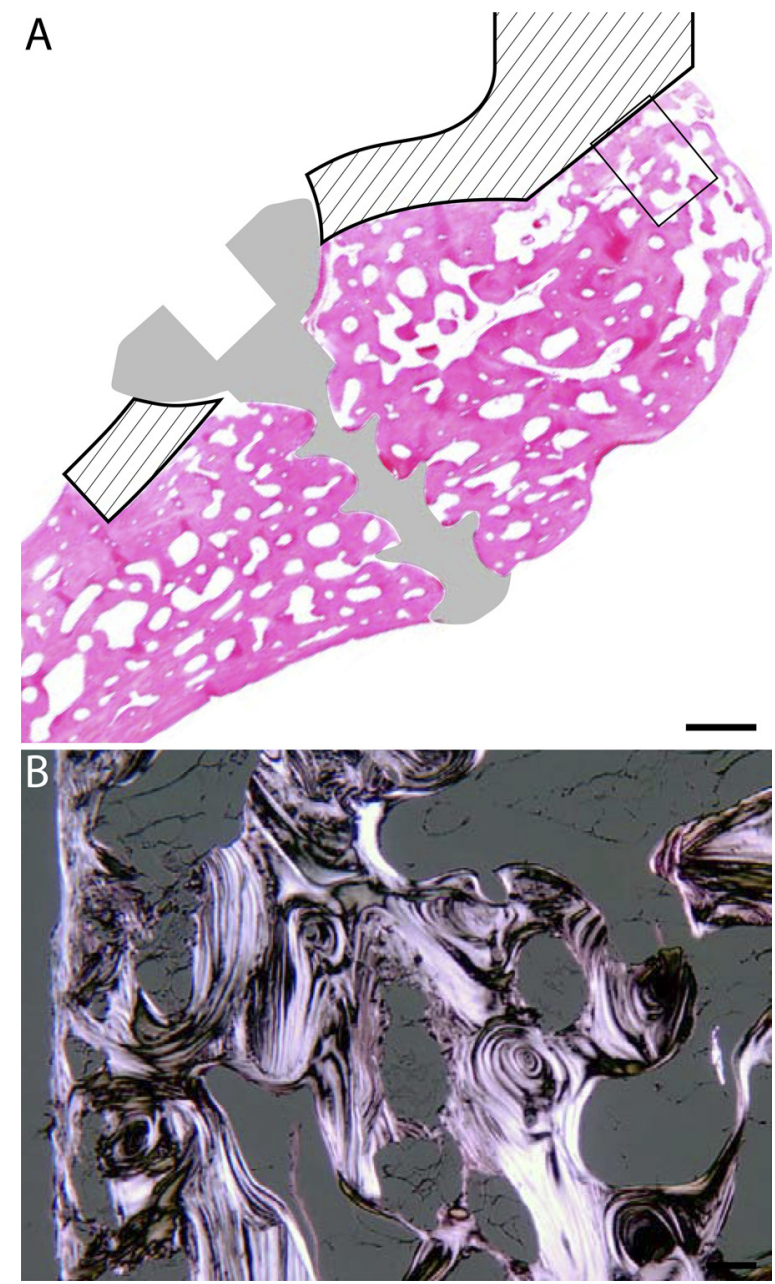

Fig. 12. Histological analysis of remodeled bone. A slice of the skull was cut from the specimen shown in Fig. 11 (between black lines in $C$ ). It was decalcified, and thin sections were cut and stained for hematoxylin and eosin. $A$ : transmitted light micrograph of the bone section (pink). The foot plate and screw cross sections have been drawn in without obscuring the tissue section. $B$ : dark-field illumination micrograph of the region outlined by the black box in A. Its histological appearance, with Haversian canals and concentric layers of lamellae, is characteristic of cortical bone. This location, under the chamber's chamfer was originally filled with hydroxyapatite paste.

2008). While chamber colonization with $S$. aureus appeared to be tolerated in our animals, it would be preferable for the chamber interior to remain sterile. For this reason, an antibiotic ointment containing neomycin, polymyxin $\mathrm{B}$, and bacitracin was placed regularly inside the recording chamber. All staphylococci species are sensitive to this triplet of topical antibiotics (Jones et al. 2006). The survey of fellow investigators revealed that triple antibiotic ointment was the most widely used agent both inside and outside recording chambers. However, different laboratories have developed a variety of successful treatment protocols and medication regimens for chamber maintenance. Laboratories should not be required to adhere to a single treatment regimen, but rather should be free to devise a system for chamber maintenance that works best in their experience.

During penetration of the dura mater, the microelectrode tip is usually withdrawn inside in a sharp hypodermic tube to protect it from damage. Once within brain tissue, the electrode can emerge safely from the protective guide tube. When recording from surface cortex located immediately under the dura, it is preferable to insert the microelectrode but not the guide tube. The dura must be thin enough to allow penetration of a bare electrode. Treatment with 5-FU arrests normal scar formation and tissue proliferation, reducing the need for dural peeling without adverse physiological or anatomical effects on cortical tissue (Spinks et al. 2003). We tested mitomycin-C as an alternative to 5-FU. It is used commonly in glaucoma surgery, where it has a similar efficacy to 5-FU (Abraham et al. 2006; WuDunn et al. 2002). Like 5-FU, mitomycin-C is a potent antibacterial agent and inhibits vascularization (Tomasz 1995). There is some evidence that mitomycin-C is longer acting and better suited for topical application than 5-FU (Anand and Khan 2009; Kim et al. 2008). However, we have not made any direct comparisons of the two agents.

In 2004, bevacizumab was introduced for the treatment of colon cancer. This antibody blocks vascular endothelial growth factor, a signaling molecule responsible for stimulating angiogenesis. We found that blocking angiogenesis in exposed dura inhibited the proliferation of granulation tissue, the precursor to fibrous scar formation. It also reduced bleeding during the thinning of dural growth. We have not compared bevacizumab with antimitotics, but a careful study would be useful to discover if there is any benefit in using one agent over the other, or even both in combination. While bevacizumab's effects on the brain have not been well studied, we have not observed any adverse effects on neuronal activity. Bevacizumab is regularly injected directly into the human eye to treat macular degeneration (CATT Research Group 2011). Some reassurance that it is safe for use in the recording chamber should be provided by its lack of deleterious effects on human retinal neurons.

The evolution of techniques is a critical factor in the advancement of any scientific field. When methodological refinements carry with them direct implications for animal welfare, it is important to pursue them. By eliminating PMMA in the implantation of devices for daily recordings in monkeys, it is possible to maintain animals in greater comfort for longer periods, thus improving their quality of life, while increasing the yield of data. However, PMMA still has advantages when multiple chambers must be placed on the skull, or when a chamber must be mounted in a stereotaxic plane to target subcortical structures.

Developments in other fields, such as material sciences and orthopedic medicine, have been valuable in guiding refinements in the field of experimental primate neurophysiology. In the 50 years since the dawn of neurophysiology in awake, behaving monkeys, improvements have been made that benefit both animals and scientific research. It is our hope that technical advances will continue to be made, aided by open sharing of collective experience.

\section{ACKNOWLEDGMENTS}

We thank colleagues in other laboratories, who have shared generously their experience with the development of titanium, acrylic-free chambers. Acknowledgement is made to the veterinarians, technicians, and husbandry staff at the California National Primate Research Center (CNPRC) and University of California, San Francisco (UCSF) for care of the animals. Vicky Cevallos at the UCSF Proctor Foundation performed the microbiological cultures. Yerem Yeghiazarians performed the fluoroscopic study. Robert H. Wurtz provided comments on the manuscript. 


\section{GRANTS}

This work was supported by the National Eye Institute (Grant R01 EY10217 and Core Grant EY02162) and Research to Prevent Blindness. Monkeys were supplied by the CNPRC, supported by National Institutes of Health Base Grant RR00169.

\section{DISCLOSURES}

No conflicts of interest, financial or otherwise, are declared by the author(s).

\section{REFERENCES}

Abraham LM, Selva D, Casson R, Leibovitch I. Mitomycin: clinical applications in ophthalmic practice. Drugs 66: 321-340, 2006.

Adams DL, Economides JR, Jocson CM, Horton JC. A biocompatible titanium headpost for stabilizing behaving monkeys. J Neurophysiol 98: 993-1001, 2007.

Anand N, Khan A. Long-term outcomes of needle revision of trabeculectomy blebs with mitomycin $\mathrm{C}$ and 5-fluorouracil: a comparative safety and efficacy report. J Glaucoma 18: 513-520, 2009.

Brånemark PI, Adell R, Breine U, Hansson BO, Lindstrom J, Ohlsson A. Intra-osseous anchorage of dental prostheses. I. Experimental studies. Scand J Plast Reconstr Surg 3: 81-100, 1969.

Buser D, Schenk RK, Steinemann S, Fiorellini JP, Fox CH, Stich H. Influence of surface characteristics on bone integration of titanium implants. A histomorphometric study in miniature pigs. J Biomed Mater Res 25: 889-902, 1991.

CATT Research Group. Ranibizumab and bevacizumab for neovascular age-related macular degeneration. N Engl J Med 364: 1897-1908, 2011.

Delgado JM. Permanent implantation of multilead electrodes in the brain. Yale J Biol Med 24: 351-358, 1952.

Evarts EV. Methods for recording activity of individual neurons in moving animals. In: Methods in Medical Research, edited by Potter VR and Rushmer RF. Chicago, IL: Year Book Medical Publishers, 1966, p. 241250.

Evarts EV. A technique for recording activity of subcortical neurons in moving animals. Electroencephalogr Clin Neurophysiol 24: 83-86, 1968.

Jones RN, Li Q, Kohut B, Biedenbach DJ, Bell J, Turnidge JD. Contemporary antimicrobial activity of triple antibiotic ointment: a multiphased study of recent clinical isolates in the United States and Australia. Diagn Microbiol Infect Dis 54: 63-71, 2006.

Kim HY, Egbert PR, Singh K. Long-term comparison of primary trabeculectomy with 5-fluorouracil versus mitomycin C in West Africa. J Glaucoma 17: 578-583, 2008.

Kleinschmidt O. Plexiglas zur Deckung von Schädellücken. Chirurgie 13: 273-277, 1941.
Kluytmans J, van Belkum A, Verbrugh H. Nasal carriage of Staphylococcus aureus: epidemiology, underlying mechanisms, and associated risks. Clin Microbiol Rev 10: 505-520, 1997.

Logothetis N, Merkle H, Augath M, Trinath T, Ugurbil K. Ultra highresolution fMRI in monkeys with implanted RF coils. Neuron 35: 227-242, 2002.

Moyad TF, Thornhill T, Estok D. Evaluation and management of the infected total hip and knee. Orthopedics 31: 581-588, 2008.

Nair S, Song Y, Meghji S, Reddi K, Harris M, Ross A, Poole S, Wilson M, Henderson B. Surface-associated proteins from Staphylococcus aureus demonstrate potent bone resorbing activity. J Bone Miner Res 10: 726-734, 1995.

Nikolis A, Malhotra G, Tiftikcioglu Y, Gupta A, Kelly C, Jackson IT. Evaluation of polymethylmethacrylate adhesion: a comparison of direct onlay versus screw anchoring techniques. J Craniomaxillofac Surg 20: 366-371, 2009.

Owens CD, Stoessel K. Surgical site infections: epidemiology, microbiology and prevention. J Hosp Infect 70, Suppl 2: 3-10, 2008.

Pohler OE. Unalloyed titanium for implants in bone surgery. Injury 31, Suppl 4: 7-13, 2000.

Rubo de Rezende ML, Johansson CB. Quantitative bone tissue response to commercially pure titanium implants. J Mater Sci Mater Med 4: 233-239, 1993.

Sanan A, Haines SJ. Repairing holes in the head: a history of cranioplasty. Neurosurgery 40: 588-603, 1997.

Sheatz GC. Electrode holders in chronic preparations. A multilead techniques for large and small animals. In: Electrical Stimulation of the Brain; An Interdisciplinary Survey of Neurobehavioral Integrative Systems, edited by Sheer DE. Austin, TX: University of Texas Press, 1961, p. 45-54.

Shi ZL, Chua PH, Neoh KG, Kang ET, Wang W. Bioactive titanium implant surfaces with bacterial inhibition and osteoblast function enhancement properties. Int J Artif Organs 31: 777-785, 2008.

Smith AW, Jackson IT, Yousefi J. The use of screw fixation of methyl methacrylate to reconstruct large craniofacial contour defects. Eur J Plast Surg 22: 17-21, 1999

Spinks RL, Baker SN, Jackson A, Khaw PT, Lemon RN. Problem of dural scarring in recording from awake, behaving monkeys: a solution using 5-fluorouracil. J Neurophysiol 90: 1324-1332, 2003.

Tomasz M. Mitomycin C: small, fast and deadly (but very selective). Chem Biol 2: 575-579, 1995.

WuDunn D, Cantor LB, Palanca-Capistrano AM, Hoop J, Alvi NP, Finley C, Lakhani V, Burnstein A, Knotts SL. A prospective randomized trial comparing intraoperative 5-fluorouracil vs. mitomycin $\mathrm{C}$ in primary trabeculectomy. Am J Ophthalmol 134: 521-528, 2002.

Wurtz RH. Visual cortex neurons: response to stimuli during rapid eye movements. Science 162: 1148-1150, 1968.

Zander E. Traitement d'urgence et réparation plastique secondaire des fractures du crâne. Z Unfallmed Berufskr 3: 222, 1963. 